\title{
Some Improvements on Hermite-Hadamard's Inequalities for $s$-convex Functions
}

\author{
Yujiao Li and Tingsong Du* \\ Department of Mathematics, Science College, China Three Gorges University, \\ Yichang 443002, Hubei, P.R. China.
}

Received 30 May 2015; Accepted (in second revised version) 15 February 2016

\begin{abstract}
Using an integral identity for a once differentiable mapping, this paper establishes Hadamard's integral inequalities for s-convex and s-concave mappings. In particular, our results improve and extend some known ones in the literature. Finally, these inequalities are applied to special means.
\end{abstract}

AMS subject classifications: 26D15, 26A51, 26E60, 41A55

Key words: Convex function, s-convex function, Hadamard's inequality.

\section{Introduction}

Throughout the present paper, we use $I \subseteq \mathbb{R}$ to denote the real interval, $I^{\circ}$ to denote the interior of $I$.

Let $f: I \subseteq \mathbb{R} \rightarrow \mathbb{R}$ be a convex function and $a, b \in I$ with $a<b$, then

$$
f\left(\frac{a+b}{2}\right) \leq \frac{1}{b-a} \int_{a}^{b} f(x) \mathrm{d} x \leq \frac{f(a)+f(b)}{2} .
$$

This remarkable result is well known in the literature as Hermite-Hadamard's inequality for convex mapping. Both inequalities hold in the reversed direction if $f$ is concave.

We know two kinds of $s$-convexity/concavity $(0<s \leq 1)$ of real valued functions are famous in the literature.

A function $f: \mathbb{R}_{+} \rightarrow \mathbb{R}$, where $\mathbb{R}_{+}=[0,+\infty)$ is said to be s-convex function in the first sense, if the inequality

$$
f(\alpha \mu+\beta v) \leq \alpha^{s} f(\mu)+\beta^{s} f(v)
$$

holds for all $\mu, v \in \mathbb{R}_{+}$, and all $\alpha, \beta \geq 0$ with $\alpha^{s}+\beta^{s}=1$.

*Corresponding author. Email addresses: yujiaolictgu@163.com (Y. J. Li), tingsongdu@ctgu.edu.cn (T. S. $\mathrm{Du})$ 
Definition 1.1. ([7]) The function $f: I \subseteq[0, \infty) \rightarrow \mathbb{R}$ is said to be s-convex function in the second sense on $I$, if the inequality

$$
f(\lambda x+(1-\lambda) y) \leq \lambda^{s} f(x)+(1-\lambda)^{s} f(y)
$$

holds for all $x, y \in I, \lambda \in[0,1]$ and for some fixed $s \in(0,1]$.

In this paper we mainly study Hadamard's integral inequalities for s-convex and sconcave mappings in the second sense. Kavurmaci et al. proved the following result connected with the right part of (1.1) in [9].

Lemma 1.1. ([9] Lemma 1) Let $f: I \subseteq \mathbb{R} \rightarrow \mathbb{R}$ be a differentiable mapping on $I^{\circ}$, where $a, b \in I$ with $a<b$. If $f^{\prime} \in L[a, b]$, then the following equality holds:

$$
\begin{aligned}
& \frac{(b-x) f(b)+(x-a) f(a)}{b-a}-\frac{1}{b-a} \int_{a}^{b} f(u) \mathrm{d} u \\
& =\frac{(x-a)^{2}}{b-a} \int_{0}^{1}(t-1) f^{\prime}(t x+(1-t) a) \mathrm{d} t+\frac{(b-x)^{2}}{b-a} \int_{0}^{1}(1-t) f^{\prime}(t x+(1-t) b) \mathrm{d} t .
\end{aligned}
$$

In recent years, a lot of inequalities of Hermite-hadamard type for convex and sconvex functions were presented, some of them can be reformulated as the following theorems.

Theorem 1.1. ([6]) Suppose that $f:[0, \infty) \rightarrow[0, \infty)$ is an s-convex function in the second sense, where $s \in(0,1]$, and let $a, b \in[0, \infty), a<b$. If $f \in L[a, b]$, then the following inequalities hold:

$$
2^{s-1} f\left(\frac{a+b}{2}\right) \leq \frac{1}{b-a} \int_{a}^{b} f(x) \mathrm{d} x \leq \frac{f(a)+f(b)}{s+1} .
$$

Theorem 1.2. ([11] Theorem 2.1) Let $f: I \subseteq \mathbb{R} \rightarrow \mathbb{R}$ be a differentiable mapping on $I^{\circ}$, where $a, b \in I^{\circ}$ with $a<b$ and let $q>1$. If $\left|f^{\prime}\right|^{q}$ is convex on $[a, b]$, then the following inequality holds:

$$
\left|f\left(\frac{a+b}{2}\right)-\frac{1}{b-a} \int_{a}^{b} f(u) \mathrm{d} u\right| \leq\left(\frac{3^{1-\frac{1}{q}}}{8}\right)(b-a)\left(\left|f^{\prime}(a)\right|+\left|f^{\prime}(b)\right|\right) .
$$

Theorem 1.3. ([1] Theorem 2.5 and [10] Theorem 2) Let $f: I \rightarrow \mathbb{R}, I \subseteq \mathbb{R}$ be a differentiable mapping on $I^{\circ}$ such that $f^{\prime} \in L[a, b]$, where $a, b \in I, a<b$. If $\left|f^{\prime}\right|^{q}$ is concave on $[a, b]$, for some fixed $q>1$, then the following inequalities hold:

$$
\left|f\left(\frac{a+b}{2}\right)-\frac{1}{b-a} \int_{a}^{b} f(u) \mathrm{d} u\right| \leq \frac{b-a}{4}\left(\frac{q-1}{2 q-1}\right)^{\frac{q-1}{q}}\left[\left|f^{\prime}\left(\frac{a+3 b}{4}\right)\right|+\left|f^{\prime}\left(\frac{3 a+b}{4}\right)\right|\right]
$$

and

$$
\left|\frac{f(a)+f(b)}{2}-\frac{1}{b-a} \int_{a}^{b} f(u) \mathrm{d} u\right| \leq \frac{b-a}{4}\left(\frac{q-1}{2 q-1}\right)^{\frac{q-1}{q}}\left[\left|f^{\prime}\left(\frac{a+3 b}{4}\right)\right|+\left|f^{\prime}\left(\frac{3 a+b}{4}\right)\right|\right] .
$$


Theorem 1.4. ([10] Theorem 3) Let $f: I \rightarrow \mathbb{R}, I \subseteq[0, \infty)$ be a differentiable mapping on $I^{\circ}$ such that $f^{\prime} \in L[a, b]$, where $a, b \in I$ with $a<b$. If $\left|f^{\prime}\right|^{q}$ is s-convex on $[a, b]$, for some fixed $s \in(0,1)$ and $q>1$, then the following inequality holds:

$$
\begin{aligned}
& \left|\frac{f(a)+f(b)}{2}-\frac{1}{b-a} \int_{a}^{b} f(u) \mathrm{d} u\right| \\
& \leq \frac{b-a}{2}\left[\left(\left|f^{\prime}(a)\right|^{q}+\left|f^{\prime}\left(\frac{a+b}{2}\right)\right|^{q}\right)^{\frac{1}{q}}+\left(\left|f^{\prime}\left(\frac{a+b}{2}\right)\right|^{q}+\left|f^{\prime}(b)\right|^{q}\right)^{\frac{1}{q}}\right] .
\end{aligned}
$$

For some recent results connected with convex, s-convex and other forms of convex, the reader can refer to $[2,3-5,8,12,14-15]$ and the references therein.

Based on our previous works [13, 16], in this paper we are going to introduce new Hadamard's integral inequalities with two parameters for a class of s-convex and sconcave functions and improve some known results in the form of corollaries.

\section{Main results}

Before proceeding towards our main theorems regarding of Hadamard type inequality for the s-convex function, we need a variant of integral identity, which is derived from Lemma 1.1.

Lemma 2.1. Let $f: I \subseteq \mathbb{R} \rightarrow \mathbb{R}$ be a differentiable mapping on $I^{\circ}$, where $a, b \in I$ with $a<b$. If $f^{\prime} \in L[a, b]$, then the following equality holds:

$$
\begin{aligned}
& (1-y) f(x)+\frac{y[(x-a) f(a)+(b-x) f(b)]}{b-a}-\frac{1}{b-a} \int_{a}^{b} f(u) \mathrm{d} u \\
& =\frac{(b-x)^{2}}{b-a} \int_{0}^{1}(y-t) f^{\prime}(t x+(1-t) b) \mathrm{d} t-\frac{(x-a)^{2}}{b-a} \int_{0}^{1}(y-t) f^{\prime}(t x+(1-t) a) \mathrm{d} t .
\end{aligned}
$$

for each $x \in(a, b)$ and $y \in[0,1]$.

Proof. We note that

$$
J=\frac{(b-x)^{2}}{b-a} \int_{0}^{1}(y-t) f^{\prime}(t x+(1-t) b) \mathrm{d} t-\frac{(x-a)^{2}}{b-a} \int_{0}^{1}(y-t) f^{\prime}(t x+(1-t) a) \mathrm{d} t
$$


Integrating by parts, we get that

$$
\begin{aligned}
J= & \frac{(b-x)^{2}}{b-a}\left[\left.\frac{y-t}{x-b} f(t x+(1-t) b)\right|_{0} ^{1}+\frac{1}{x-b} \int_{0}^{1} f(t x+(1-t) b) \mathrm{d} t\right] \\
& -\frac{(x-a)^{2}}{b-a}\left[\left.\frac{y-t}{x-a} f(t x+(1-t) a)\right|_{0} ^{1}+\frac{1}{x-a} \int_{0}^{1} f(t x+(1-t) a) \mathrm{d} t\right] \\
= & \frac{(b-x)^{2}}{b-a}\left[\frac{y-1}{x-b} f(x)-\frac{y}{x-b} f(b)+\frac{1}{(x-b)^{2}} \int_{b}^{x} f(u) \mathrm{d} u\right] \\
& -\frac{(x-a)^{2}}{b-a}\left[\frac{y-1}{x-a} f(x)-\frac{y}{x-a} f(a)+\frac{1}{(x-a)^{2}} \int_{a}^{x} f(u) \mathrm{d} u\right] \\
= & (1-y) f(x)+\frac{y[(x-a) f(a)+(b-x) f(b)]}{b-a}-\frac{1}{b-a} \int_{a}^{b} f(u) \mathrm{d} u .
\end{aligned}
$$

Lemma 2.1 is thus proved.

Remark 2.1. Under the conditions of Lemma 2.1, if we take $y=1$, then Lemma 2.1 coincides with Lemma 1 established by Kavurmaci et al. in [9].

Now, the main results about Hadamard's integral inequalities for s-convex and sconcave mappings will be presented.

Theorem 2.1. Let $f: I \subseteq \mathbb{R}_{0} \rightarrow \mathbb{R}$ be a differentiable mapping on $I^{\circ}$ such that $f^{\prime} \in L[a, b]$, where $a, b \in I$ with $a<b$. If $\left|f^{\prime}\right|^{q}$ is s-convex on $[a, b], q>1, p=\frac{q}{q-1}, s \in(0,1]$, and for each $x \in[a, b]$ and $y \in[0,1]$, then the following inequality holds:

$$
\begin{aligned}
& \left|(1-y) f(x)+\frac{y[(x-a) f(a)+(b-x) f(b)]}{b-a}-\frac{1}{b-a} \int_{a}^{b} f(u) \mathrm{d} u\right| \\
& \leq\left(y^{2}-y+\frac{1}{2}\right)^{\frac{q-1}{q}}\left[\frac{(x-a)^{2}}{b-a}\left(K\left|f^{\prime}(a)\right|^{q}+T\left|f^{\prime}(x)\right|^{q}\right)^{\frac{1}{q}}+\frac{(b-x)^{2}}{b-a}\left(T\left|f^{\prime}(x)\right|^{q}+K\left|f^{\prime}(b)\right|^{q}\right)^{\frac{1}{q}}\right],
\end{aligned}
$$

where $K=\frac{2(1-y)^{s+2}+y(s+2)-1}{(s+1)(s+2)}$ and $T=\frac{2 y^{s+2}-y(s+2)+s+1}{(s+1)(s+2)}$.

Proof. From Lemma 2.1, it follows that

$$
\begin{aligned}
& \left|(1-y) f(x)+\frac{y[(x-a) f(a)+(b-x) f(b)]}{b-a}-\frac{1}{b-a} \int_{a}^{b} f(u) \mathrm{d} u\right| \\
& \leq \frac{(x-a)^{2}}{b-a} \int_{0}^{1}|y-t|\left|f^{\prime}(t x+(1-t) a)\right| \mathrm{d} t+\frac{(b-x)^{2}}{b-a} \int_{0}^{1}|y-t|\left|f^{\prime}(t x+(1-t) b)\right| \mathrm{d} t .
\end{aligned}
$$


Using well known power-mean inequality, for $q>1$ and $p=\frac{q}{q-1}$, it yields that

$$
\begin{aligned}
\int_{0}^{1}|y-t|\left|f^{\prime}(t x+(1-t) a)\right| \mathrm{d} t & =\int_{0}^{1}|y-t|^{1-\frac{1}{q}}|y-t|^{\frac{1}{q}}\left|f^{\prime}(t x+(1-t) a)\right| \mathrm{d} t \\
& \leq\left(\int_{0}^{1}|y-t| \mathrm{d} t\right)^{\frac{q-1}{q}}\left(\int_{0}^{1}|y-t|\left|f^{\prime}(t x+(1-t) a)\right|^{q} \mathrm{~d} t\right)^{\frac{1}{q}}
\end{aligned}
$$

and

$$
\int_{0}^{1}|y-t|\left|f^{\prime}(t x+(1-t) b)\right| \mathrm{d} t \leq\left(\int_{0}^{1}|y-t| \mathrm{d} t\right)^{\frac{q-1}{q}}\left(\int_{0}^{1}|y-t|\left|f^{\prime}(t x+(1-t) b)\right|^{q} \mathrm{~d} t\right)^{\frac{1}{q}} .
$$

Since $\left|f^{\prime}\right|^{q}$ is s-convex on $[a, b]$, we know that for every $t \in[0,1]$

$$
\left|f^{\prime}(t a+(1-t) b)\right|^{q} \leq t^{s}\left|f^{\prime}(a)\right|^{q}+(1-t)^{s}\left|f^{\prime}(b)\right|^{q}
$$

and thus, we obtain

$$
\begin{aligned}
& \left|(1-y) f(x)+\frac{y[(x-a) f(a)+(b-x) f(b)]}{b-a}-\frac{1}{b-a} \int_{a}^{b} f(u) \mathrm{d} u\right| \\
& \leq \frac{(x-a)^{2}}{b-a}\left[\left(\int_{0}^{1}|y-t| \mathrm{d} t\right)^{\frac{q-1}{q}}\left(\int_{0}^{1}|y-t|\left((1-t)^{s}\left|f^{\prime}(a)\right|^{q}+t^{s}\left|f^{\prime}(x)\right|^{q}\right) \mathrm{d} t\right)^{\frac{1}{q}}\right] \\
& \quad+\frac{(b-x)^{2}}{b-a}\left[\left(\int_{0}^{1}|y-t| \mathrm{d} t\right)^{\frac{q-1}{q}}\left(\int_{0}^{1}|y-t|\left(t^{s}\left|f^{\prime}(x)\right|^{q}+(1-t)^{s}\left|f^{\prime}(b)\right|^{q}\right) \mathrm{d} t\right)^{\frac{1}{q}}\right] \\
& =\left[\int_{0}^{1}|y-t| \mathrm{d} t\right]^{\frac{q-1}{q}}\left\{\frac{(x-a)^{2}}{b-a}\left[\int_{0}^{1}|y-t|\left((1-t)^{s}\left|f^{\prime}(a)\right|^{q}+t^{s}\left|f^{\prime}(x)\right|^{q}\right) \mathrm{d} t\right]^{\frac{1}{q}}\right. \\
& \left.\quad+\frac{(b-x)^{2}}{b-a}\left[\int_{0}^{1}|y-t|\left(t^{s}\left|f^{\prime}(x)\right|^{q}+(1-t)^{s}\left|f^{\prime}(b)\right|^{q}\right) \mathrm{d} t\right]^{\frac{1}{q}}\right\} .
\end{aligned}
$$

Using the fact that

$$
\int_{0}^{1}|y-t|(1-t)^{s} \mathrm{~d} t=\frac{2(1-y)^{s+2}+y(s+2)-1}{(s+1)(s+2)}, \int_{0}^{1}|y-t| t^{s} \mathrm{~d} t=\frac{2 y^{s+2}-y(s+2)+s+1}{(s+1)(s+2)} .
$$

We get the required result.

Corollary 2.1. Under the conditions of Theorem 2.1, 
(1) if we choose $x=\frac{a+b}{2}, y=0$, and $s=1$, we have

$$
\begin{aligned}
& \left|f\left(\frac{a+b}{2}\right)-\frac{1}{b-a} \int_{a}^{b} f(u) \mathrm{d} u\right| \\
& \leq \frac{b-a}{4}\left(\frac{1}{2}\right)^{\frac{q-1}{q}}\left[\left(\frac{2\left|f^{\prime}\left(\frac{a+b}{2}\right)\right|^{q}+\left|f^{\prime}(a)\right|^{q}}{6}\right)^{\frac{1}{q}}+\left(\frac{2\left|f^{\prime}\left(\frac{a+b}{2}\right)\right|^{q}+\left|f^{\prime}(b)\right|^{q}}{6}\right)^{\frac{1}{q}}\right] ;
\end{aligned}
$$

(2) if we choose $x=\frac{a+b}{2}, y=1$, and $s=1$, we also obtain

$$
\begin{aligned}
& \left|\frac{f(a)+f(b)}{2}-\frac{1}{b-a} \int_{a}^{b} f(u) \mathrm{d} u\right| \\
& \leq \frac{b-a}{4}\left(\frac{1}{2}\right)^{\frac{q-1}{q}}\left[\left(\frac{\left|f^{\prime}\left(\frac{a+b}{2}\right)\right|^{q}+2\left|f^{\prime}(a)\right|^{q}}{6}\right)^{\frac{1}{q}}+\left(\frac{\left|f^{\prime}\left(\frac{a+b}{2}\right)\right|^{q}+2\left|f^{\prime}(b)\right|^{q}}{6}\right)^{\frac{1}{q}}\right] .
\end{aligned}
$$

Corollary 2.2. Under the conditions of Corollary 2.1 (1), using the fact $\sum_{i=1}^{n}\left(a_{i}+b_{i}\right)^{r} \leq$ $\sum_{i=1}^{n} a_{i}^{r}+\sum_{i=1}^{n} b_{i}^{r}$, for $0<r<1, a_{1}, \ldots, a_{n} \geq 0$ and $b_{1}, \ldots, b_{n} \geq 0$. Then using the convexity of $|f|^{\prime}$, we obtain

$$
\begin{aligned}
& \left|f\left(\frac{a+b}{2}\right)-\frac{1}{b-a} \int_{a}^{b} f(u) \mathrm{d} u\right| \\
& \leq \frac{b-a}{4}\left(\frac{1}{2}\right)^{\frac{q-1}{q}}\left(\frac{1}{6}\right)^{\frac{1}{q}}\left[\left(2\left|f^{\prime}\left(\frac{a+b}{2}\right)\right|^{q}+\left|f^{\prime}(a)\right|^{q}\right)^{\frac{1}{q}}+\left(2\left|f^{\prime}\left(\frac{a+b}{2}\right)\right|^{q}+\left|f^{\prime}(b)\right|^{q}\right)^{\frac{1}{q}}\right] \\
& \leq \frac{b-a}{8}\left(\frac{1}{3}\right)^{\frac{1}{q}}\left(1+2^{\frac{1}{q}}\right)\left(\left|f^{\prime}(a)\right|+\left|f^{\prime}(b)\right|\right) \\
& \leq\left(\frac{3^{1-\frac{1}{q}}}{8}\right)(b-a)\left(\left|f^{\prime}(a)\right|+\left|f^{\prime}(b)\right|\right),
\end{aligned}
$$

which is an improved result comparing with inequality of (1.5). And the inequality of (1.5) is just Theorem 2.1 established by Kirmaci and Özdemir in [11].

Theorem 2.2. Let $f: I \subseteq \mathbb{R}_{0} \rightarrow \mathbb{R}$ be a differentiable mapping on $I^{\circ}$ such that $f^{\prime} \in L[a, b]$, where $a, b \in I$ with $a<b$. If $\left|f^{\prime}\right|^{q}$ is s-convex on $[a, b]$ and $q>1$, for each $x \in[a, b], y \in[0,1]$ and some fixed $s \in(0,1]$, then the following inequality holds:

$$
\begin{aligned}
& \left|(1-y) f(x)+\frac{y[(x-a) f(a)+(b-x) f(b)]}{b-a}-\frac{1}{b-a} \int_{a}^{b} f(u) \mathrm{d} u\right| \\
& \leq\left(\frac{q-1}{2 q-1}\right)^{\frac{q-1}{q}}\left(\frac{1}{s+1}\right)^{\frac{1}{q}}\left(y^{\frac{2 q-1}{q-1}}+(1-y)^{\frac{2 q-1}{q-1}}\right)^{\frac{q-1}{q}} \\
& \quad \times\left[\frac{(x-a)^{2}\left(\left|f^{\prime}(a)\right|^{q}+\left|f^{\prime}(x)\right|^{q}\right)^{\frac{1}{q}}+(b-x)^{2}\left(\left|f^{\prime}(x)\right|^{q}+\left|f^{\prime}(b)\right|^{q}\right)^{\frac{1}{q}}}{b-a}\right] .
\end{aligned}
$$


Proof. By Lemma 2.1, using the famous Hölder integral inequality in the following way, we get

$$
\begin{aligned}
& \left|(1-y) f(x)+\frac{y[(x-a) f(a)+(b-x) f(b)]}{b-a}-\frac{1}{b-a} \int_{a}^{b} f(u) \mathrm{d} u\right| \\
& \leq \frac{(x-a)^{2}}{b-a} \int_{0}^{1}|y-t|\left|f^{\prime}(t x+(1-t) a)\right| \mathrm{d} t+\frac{(b-x)^{2}}{b-a} \int_{0}^{1}|y-t|\left|f^{\prime}(t x+(1-t) b)\right| \mathrm{d} t \\
& \leq \frac{(x-a)^{2}}{b-a}\left[\left(\int_{0}^{1}|y-t|^{\frac{q}{q-1}} \mathrm{~d} t\right)^{\frac{q-1}{q}}\left(\int_{0}^{1}\left|f^{\prime}(t x+(1-t) a)\right|^{q} \mathrm{~d} t\right)^{\frac{1}{q}}\right] \\
& \quad+\frac{(b-x)^{2}}{b-a}\left[\left(\int_{0}^{1}|y-t|^{\frac{q}{q-1}} \mathrm{~d} t\right)^{\frac{q-1}{q}}\left(\int_{0}^{1}\left|f^{\prime}(t x+(1-t) b)\right|^{q} \mathrm{~d} t\right)^{\frac{1}{q}}\right] .
\end{aligned}
$$

From inequality (1.4), the following inequalities can be easily obtained

$$
\begin{gathered}
\int_{0}^{1}\left|f^{\prime}(t x+(1-t) a)\right|^{q} \mathrm{~d} t=\frac{1}{x-a} \int_{a}^{x}\left|f^{\prime}(u)\right|^{q} \mathrm{~d} u \leq \frac{1}{s+1}\left(\left|f^{\prime}(a)\right|^{q}+\left|f^{\prime}(x)\right|^{q}\right), \\
\int_{0}^{1}\left|f^{\prime}(t x+(1-t) b)\right|^{q} \mathrm{~d} t \leq \frac{1}{s+1}\left(\left|f^{\prime}(x)\right|^{q}+\left|f^{\prime}(b)\right|^{q}\right) .
\end{gathered}
$$

Then, we have

$$
\begin{aligned}
& \left|(1-y) f(x)+\frac{y[(x-a) f(a)+(b-x) f(b)]}{b-a}-\frac{1}{b-a} \int_{a}^{b} f(u) \mathrm{d} u\right| \\
& \leq \frac{(x-a)^{2}}{b-a}\left[\left(\frac{q-1}{2 q-1}\left(y^{\frac{2 q-1}{q-1}}+(1-y)^{\frac{2 q-1}{q-1}}\right)\right)^{\frac{q-1}{q}}\left(\frac{1}{s+1}\right)^{\frac{1}{q}}\left(\left|f^{\prime}(a)\right|^{q}+\left|f^{\prime}(x)\right|^{q}\right)^{\frac{1}{q}}\right] \\
& \quad+\frac{(b-x)^{2}}{b-a}\left[\left(\frac{q-1}{2 q-1}\left(y^{\frac{2 q-1}{q-1}}+(1-y)^{\frac{2 q-1}{q-1}}\right)\right)^{\frac{q-1}{q}}\left(\frac{1}{s+1}\right)^{\frac{1}{q}}\left(\left|f^{\prime}(x)\right|^{q}+\left|f^{\prime}(b)\right|^{q}\right)^{\frac{1}{q}}\right] \\
& =\left(\frac{q-1}{2 q-1}\right)^{\frac{q-1}{q}}\left(\frac{1}{s+1}\right)^{\frac{1}{q}}\left(y^{\frac{2 q-1}{q-1}}+(1-y)^{\frac{2 q-1}{q-1}}\right)^{\frac{q-1}{q}} \\
& \quad \times\left[\frac{(x-a)^{2}\left(\left|f^{\prime}(a)\right|^{q}+\left|f^{\prime}(x)\right|^{q}\right)^{\frac{1}{q}}+(b-x)^{2}\left(\left|f^{\prime}(x)\right|^{q}+\left|f^{\prime}(b)\right|^{q}\right)^{\frac{1}{q}}}{b-a}\right] .
\end{aligned}
$$

This completes the proof of Theorem 2.2.

Corollary 2.3. Under the conditions of Theorem 2.2, 
(1) if we choose $x=\frac{a+b}{2}, y=0$, and $s=1$, we obtain

$$
\begin{aligned}
& \left|f\left(\frac{a+b}{2}\right)-\frac{1}{b-a} \int_{a}^{b} f(u) \mathrm{d} u\right| \\
& \leq\left(\frac{q-1}{2 q-1}\right)^{\frac{q-1}{q}}\left(\frac{1}{2}\right)^{\frac{1}{q}}\left(\frac{b-a}{4}\right)\left[\left(\left|f^{\prime}(a)\right|^{q}+\left|f^{\prime}\left(\frac{a+b}{2}\right)\right|^{q}\right)^{\frac{1}{q}}+\left(\left|f^{\prime}\left(\frac{a+b}{2}\right)\right|^{q}+\left|f^{\prime}(b)\right|^{q}\right)^{\frac{1}{q}}\right] \\
& \leq\left(\frac{b-a}{2}\right)\left[\left(\left|f^{\prime}(a)\right|^{q}+\left|f^{\prime}\left(\frac{a+b}{2}\right)\right|^{q}\right)^{\frac{1}{q}}+\left(\left|f^{\prime}\left(\frac{a+b}{2}\right)\right|^{q}+\left|f^{\prime}(b)\right|^{q}\right)^{\frac{1}{q}}\right] ;
\end{aligned}
$$

(2) if we choose $x=\frac{a+b}{2}, y=1$, and $s=1$, we have

$$
\begin{aligned}
& \left|\frac{f(a)+f(b)}{2}-\frac{1}{b-a} \int_{a}^{b} f(u) \mathrm{d} u\right| \\
& \leq\left(\frac{q-1}{2 q-1}\right)^{\frac{q-1}{q}}\left(\frac{1}{2}\right)^{\frac{1}{q}}\left(\frac{b-a}{4}\right)\left[\left(\left|f^{\prime}(a)\right|^{q}+\left|f^{\prime}\left(\frac{a+b}{2}\right)\right|^{q}\right)^{\frac{1}{q}}+\left(\left|f^{\prime}\left(\frac{a+b}{2}\right)\right|^{q}+\left|f^{\prime}(b)\right|^{q}\right)^{\frac{1}{q}}\right] \\
& \leq\left(\frac{b-a}{2}\right)\left[\left(\left|f^{\prime}(a)\right|^{q}+\left|f^{\prime}\left(\frac{a+b}{2}\right)\right|^{q}\right)^{\frac{1}{q}}+\left(\left|f^{\prime}\left(\frac{a+b}{2}\right)\right|^{q}+\left|f^{\prime}(b)\right|^{q}\right)^{\frac{1}{q}}\right] .
\end{aligned}
$$

It is noted that the result of the first inequality (2.7) is better than the inequality (1.8) presented by Kiramic et al. in [10].

Theorem 2.3. Let $f: I \subseteq \mathbb{R}_{0} \rightarrow \mathbb{R}$ be a differentiable mapping on $I^{\circ}$ such that $f^{\prime} \in L[a, b]$, where $a, b \in I$ with $a<b$. If $\left|f^{\prime}\right|^{q}$ is s-concave on $[a, b]$, for each $x \in[a, b], y \in[0,1]$ and some fixed $s \in(0,1)$ and $q>1$, then:

$$
\begin{aligned}
& \left|(1-y) f(x)+\frac{y[(x-a) f(a)+(b-x) f(b)]}{b-a}-\frac{1}{b-a} \int_{a}^{b} f(u) \mathrm{d} u\right| \\
& \leq 2^{\frac{s-1}{q}}\left(\frac{q-1}{2 q-1}\right)^{\frac{q-1}{q}}\left(y^{\frac{2 q-1}{q-1}}+(1-y)^{\frac{2 q-1}{q-1}}\right)^{\frac{q-1}{q}}\left[\frac{(x-a)^{2}\left|f^{\prime}\left(\frac{x+a}{2}\right)\right|+(b-x)^{2}\left|f^{\prime}\left(\frac{x+b}{2}\right)\right|}{b-a}\right] .
\end{aligned}
$$

Proof. We proceed similarly as in the proof of Theorem 2.2, the only difference is that we use the s-concavity of $\left|f^{\prime}\right|^{q}$ and inequality (1.4), then

$$
\int_{0}^{1}\left|f^{\prime}(t x+(1-t) a)\right|^{q} \mathrm{~d} t=\frac{1}{x-a} \int_{a}^{x}\left|f^{\prime}(u)\right|^{q} \mathrm{~d} u \leq 2^{s-1}\left|f^{\prime}\left(\frac{x+a}{2}\right)\right|^{q},
$$

similarly,

$$
\int_{0}^{1}\left|f^{\prime}(t x+(1-t) b)\right|^{q} \mathrm{~d} t \leq 2^{s-1}\left|f^{\prime}\left(\frac{x+b}{2}\right)\right|^{q}
$$


So, we can show that

$$
\begin{aligned}
\mid & (1-y) f(x)+\frac{y[(x-a) f(a)+(b-x) f(b)]}{b-a}-\frac{1}{b-a} \int_{a}^{b} f(u) \mathrm{d} u \mid \\
\leq \leq & \frac{(x-a)^{2}}{b-a}\left[\left(\int_{0}^{1}|y-t|^{\frac{q}{q-1}} \mathrm{~d} t\right)^{\frac{q-1}{q}}\left(\int_{0}^{1}\left|f^{\prime}(t x+(1-t) a)\right|^{q} \mathrm{~d} t\right)^{\frac{1}{q}}\right] \\
& +\frac{(b-x)^{2}}{b-a}\left[\left(\int_{0}^{1}|y-t|^{\frac{q}{q-1}} \mathrm{~d} t\right)^{\frac{q-1}{q}}\left(\int_{0}^{1}\left|f^{\prime}(t x+(1-t) b)\right|^{q} \mathrm{~d} t\right)^{\frac{1}{q}}\right] \\
\leq & \frac{(x-a)^{2}}{b-a}\left[\frac{q-1}{2 q-1}\left(y^{\frac{2 q-1}{q-1}}+(1-y)^{\frac{2 q-1}{q-1}}\right)\right]^{\frac{q-1}{q}} 2^{\frac{s-1}{q}}\left|f^{\prime}\left(\frac{x+a}{2}\right)\right| \\
& +\frac{(b-x)^{2}}{b-a}\left[\frac{q-1}{2 q-1}\left(y^{\frac{2 q-1}{q-1}}+(1-y)^{\frac{2 q-1}{q-1}}\right)\right]^{\frac{q-1}{q}} 2^{\frac{s-1}{q}}\left|f^{\prime}\left(\frac{x+b}{2}\right)\right| \\
= & 2^{\frac{s-1}{q}}\left(\frac{q-1}{2 q-1}\right)^{\frac{q-1}{q}}\left(y^{\frac{2 q-1}{q-1}}+(1-y)^{\frac{2 q-1}{q-1}}\right)^{\frac{q-1}{q}}\left[\frac{(x-a)^{2}\left|f^{\prime}\left(\frac{x+a}{2}\right)\right|+(b-x)^{2}\left|f^{\prime}\left(\frac{x+b}{2}\right)\right|}{b-a}\right],
\end{aligned}
$$

which completes the proof.

Corollary 2.4. Under the conditions of Theorem 2.3,

(1) if we choose $x=\frac{a+b}{2}$ and $y=0$, we have

$$
\begin{aligned}
\left|f\left(\frac{a+b}{2}\right)-\frac{1}{b-a} \int_{a}^{b} f(u) \mathrm{d} u\right| & \leq\left(\frac{q-1}{2 q-1}\right)^{\frac{q-1}{q}}\left(\frac{b-a}{4}\right) 2^{\frac{s-1}{q}}\left(\left|f^{\prime}\left(\frac{3 a+b}{4}\right)\right|+\left|f^{\prime}\left(\frac{a+3 b}{4}\right)\right|\right) \\
& \leq\left(\frac{q-1}{2 q-1}\right)^{\frac{q-1}{q}}\left(\frac{b-a}{4}\right)\left(\left|f^{\prime}\left(\frac{3 a+b}{4}\right)\right|+\left|f^{\prime}\left(\frac{a+3 b}{4}\right)\right|\right)
\end{aligned}
$$

(2) if we choose $x=\frac{a+b}{2}$ and $y=1$, we have

$$
\begin{aligned}
\left|\frac{f(a)+f(b)}{2}-\frac{1}{b-a} \int_{a}^{b} f(u) \mathrm{d} u\right| & \leq\left(\frac{q-1}{2 q-1}\right)^{\frac{q-1}{q}}\left(\frac{b-a}{4}\right) 2^{\frac{s-1}{q}}\left(\left|f^{\prime}\left(\frac{3 a+b}{4}\right)\right|+\left|f^{\prime}\left(\frac{a+3 b}{4}\right)\right|\right) \\
& \leq\left(\frac{q-1}{2 q-1}\right)^{\frac{q-1}{q}}\left(\frac{b-a}{4}\right)\left(\left|f^{\prime}\left(\frac{3 a+b}{4}\right)\right|+\left|f^{\prime}\left(\frac{a+3 b}{4}\right)\right|\right) .
\end{aligned}
$$

It is observed that the first inequality of inequalities 2.9 and 2.10 give an improvement of inequalities 1.6 and 1.7 established by Alomari and Kirmaci, respectively.

\section{Applications to special means}

For positive numbers, $\beta>\alpha>0$ and $n \in \mathbb{Z} \backslash\{0,-1\}$, define 


$$
A(\alpha, \beta)=\frac{\alpha+\beta}{2}, L(\alpha, \beta)=\frac{\alpha-\beta}{\ln |\alpha|-\ln |\beta|}, L_{n}(\alpha, \beta)=\left[\frac{\beta^{n+1}-\alpha^{n+1}}{(n+1)(\beta-\alpha)}\right]^{\frac{1}{n}} \text {. }
$$

These quantities are respectively called the arithmetic, logarithmic and generalized logarithmic means of two positive number $\alpha$ and $\beta$.

Now, we give some applications to special means of real numbers using the results of Section 2.

Proposition 3.1. Let $a<b, 0<s<1$, we have

$$
\begin{aligned}
& \left|A\left(a^{s}, b^{s}\right)-L_{s}^{s}(a, b)\right| \\
& \leq s\left(\frac{b-a}{4}\right)\left(\frac{1}{2}\right)^{\frac{q-1}{q}}\left(\frac{1}{s+2}\right)^{\frac{1}{q}}\left\{\left[|a|^{q(s-1)}+\frac{1}{s+1}|x|^{q(s-1)}\right]^{\frac{1}{q}}+\left[|x|^{q(s-1)}+\frac{1}{s+1}|b|^{q(s-1)}\right]^{\frac{1}{q}}\right\} .
\end{aligned}
$$

Proof. When we choose $x=\frac{a+b}{2}, y=1$, the inequality (3.1) follows from (2.2) applied to the $s$-convex function in the second sense $f:[0,1] \rightarrow[0,1], f(t)=t^{s}$. The details are omitted.

Proposition 3.2. Let $a<b, 0<s<1$, for all $q>1$, we have

$$
\begin{aligned}
& \left|A\left(a^{s}, b^{s}\right)-L_{s}^{s}(a, b)\right| \\
& \leq s\left(\frac{b-a}{4}\right)\left(\frac{1}{s+1}\right)^{\frac{1}{q}}\left(\frac{q-1}{2 q-1}\right)^{\frac{q-1}{q}}\left[\left(|a|^{q(s-1)}+|x|^{q(s-1)}\right)^{\frac{1}{q}}+\left(|x|^{q(s-1)}+|b|^{q(s-1)}\right)^{\frac{1}{q}}\right] .
\end{aligned}
$$

Proof. When we choose $x=\frac{a+b}{2}, y=1$, the proof is similar to that of Proposition 3.1, using Theorem 2.2.

Proposition 3.3. Let $a<b, 0<s<1$, for all $q>1$, we have

$$
\left|A\left(a^{-1}, b^{-1}\right)-L^{-1}(a, b)\right| \leq 2^{\frac{s-1}{q}}\left(\frac{b-a}{4}\right)\left(\frac{q-1}{2 q-1}\right)^{\frac{q-1}{q}}\left[\left|\frac{3 a+b}{4}\right|^{-2 q}+\left|\frac{a+3 b}{4}\right|^{-2 q}\right] .
$$

Proof. When we choose $x=\frac{a+b}{2}, y=1$, the inequality (3.3) follows from (2.10) applied to the $s$-convex function in the second sense $f:[0,1] \rightarrow[0,1], f(t)=\frac{1}{t}$. The details are omitted.

\section{Acknowledgements}

This work was supported by NSFC (Grant No. 61374028), Hubei Province Key Laboratory of Systems Science in Metallurgical Process of China (Grant No. Z201402), and the Natural Science Foundation of Hubei Province (Grant No. 2013CFA131). The authors sincerely appreciate the reviewers for their careful reading and significant comments which have resulted in the present improved version of the original paper. 


\section{References}

[1] M.W. Alomari, M. Darus, U.S. Kirmaci. Some inequalities of Hermite-Hadamard type for s-convex functions. Acta Math. Sci., 2011, 31B(4): 1643-1652.

[2] M. Avci, H. Kavurmaci, M.E. Özdemir. New inequalities of Hermite-Hadamard type via s-convex functions in the second sense with applications. Appl. Math. Comput., 2011, 217: 5171-5176.

[3] F.-X. Chen, Y.-M. Feng. New inequalities of Hermite-Hadamard type for functions whose first derivatives absolute values are s-convex. Italian J. Pure Appl. Math., 2014, 32: 213-222.

[4] L. Chun, F. Qi. Integral inequalities of Hermite-Hadamard type for functions whose third derivatives are convex. J. Inequal. Appl., 2013, 451: 10 pages, available online at http:/ /dx.doi.org/10.1186/1029-242X-2013-451.

[5] S.S. Dragomir, R.P. Agarwal. Two inequalities for differentiable mappings and applications to special means of real numbers and to trapezoidal formula. Appl. Math. Lett., 1998, 11(5): 91-95.

[6] S.S. Dragomir, S. Fitzpatrick. The Hadamard's inequality for s-convex functions in the second sense. Demonstratio Math., 1999, 32(4): 687-696.

[7] H. Hudzik, L. Maligranda. Some remarks on s-convex functions. Aequationes Math., 1994, 48: 100-111.

[8] U.S. Kirmaci. Inequalities for differentiable mappings and applications to special means of real numbers and to midpoint formula. Appl. Math. Comput., 2004, 147: 137-146.

[9] H. Kavurmaci, M. Avci, M.E. Özdemir. New inequalities of Hermite-Hadamard for convex functions with applications. J. Inequal. Appl., 2011, 86: 11 pages, available online at http:/ / dx.doi.org/10.1186/1029-242X-2011-86.

[10] U.S. Kirmaci, M.K. Bakula, M.E. Özdemir, J. Pečarić. Hadamard-type inequalities for sconvex functions. Appl. Math. Comput., 2007, 193(1): 26-35.

[11] U.S. Kirmaci, M.E. Özdemir. On some inequalities for differentiable mappings and applications to special means of real numbers and to midpoint formula. Appl. Math. Comput., 2004, 153(2): 361-368.

[12] M.A. Latif. Inequalities of Hermite-Hadamard type for functions whose derivatives in absolute value are convex with applications. Arab J. Math. Sci., 2014, 21(1): 84-97.

[13] Y.-J. Li, T.-S. Du. On Simpson type inequalities for functions whose derivatives are extended ( $s, m)$-GA-convex functions. Pure Appl. Math., 2015, 31(5): 487-497.

[14] M.E. Özdemir, Ç. Yıldız, A.O. Akdemir, E. Set. On some inequalities for s-convex functions and applications. J. Inequal. Appl., 2013, 333: 11 pages, available online at http:/ /dx.doi.org/10.1186/1029-242X-2013-333.

[15] M. Tunç. Some Hadamard-like inequalities via convex and s-convex functions and their Applications for Special Means. Mediterr. J. Math., 2014, 11: 1047-1059.

[16] Z.-Q. Yang, Y.-J. Li, T.-S. Du. A generalization of Simpson type inequality via differentiable functions using $(s, m)$-convex functions. Italian J. Pure Appl. Math., 2015, 35: 327-338. 